\title{
ANÁLISE ESPACIAL DA PRECIPITAÇÃO PLUVIOMÉTRICA NO ESTADO DE SÃO PAULO: COMPARAÇÃO DE MÉTODOS DE INTERPOLAÇÃ̃ ${ }^{1}$
}

\author{
JOSÉ R. P. DE CARVALHO², EDUARDO D. ASSAD ${ }^{3}$
}

\begin{abstract}
RESUMO: O objetivo deste trabalho é comparar três interpoladores univariados: inverso do quadrado da distância, curvatura mínima e krigagem ordinária usando observações de precipitação pluvial média anual de 1.027 postos pluviométricos abrangendo todo o Estado de São Paulo, representando área de $248.808,8 \mathrm{~km}^{2}$ (2,91\% do território nacional), no período de 1957 a 1997. Os mapas de variabilidade espacial não apresentaram grandes diferenças visuais; entretanto, quando a estatística quadrado médio do erro foi calculada para cada técnica, confirmou-se a grande vantagem em usar o interpolador ótimo de krigagem. A diferença entre os valores observados e estimados pela krigagem foi muito menor do que para os outros dois métodos, indicando ser esse o interpolador a ser usado na distribuição espacial de precipitação pluvial média anual para os dados em estudo.
\end{abstract}

PALAVRAS-CHAVE: krigagem ordinária, geoestatística, semivariograma.

\section{SPATIAL ANALYSIS OF PRECIPITATION DATA IN SÃO PAULO STATE: COMPARISON OF INTERPOLATION METHODS}

\begin{abstract}
The objective of this paper is to compare three unvaried interpolation algorithms: inverse square distance, minimum curvature and ordinary kriging using observations of annual precipitation average of one thousand and twenty-seven climatic stations including all State of São Paulo, representing an area of approximately $248,808.8 \mathrm{~km}^{2}$ (2,91\% of the brazilian territory), from 1957 to 1997 . The maps of spatial variability didn't present great visual differences, however when the comparison criterion, the mean square error of prediction was calculated for each technique, the great advantage was confirmed by using kriging interpolator. The difference among the observed and predicted values for kriging was much smaller than for the other two methods, indicating to be this the interpolator to be used in the spatial distribution of annual precipitation for this data.
\end{abstract}

KEYWORDS: ordinary kriging, geostatistic, semivariogram.

\section{INTRODUÇÃO}

Medir dados de precipitação pluvial é muito importante em diversos contextos, tais como, produção da cultura, manejo dos recursos hídricos, avaliação ambiental, erosão hídrica, etc. A obtenção da correta distribuição espacial para precipitação é relevante no planejamento agrícola, no que diz respeito à instalação de culturas. Além da influência na agricultura, períodos de estiagens muito longos afetam o nível de água dos mananciais e dos reservatórios das usinas hidrelétricas, trazendo problemas para o abastecimento urbano e na geração de energia elétrica. A quantificação das chuvas com intensidades superiores ao suporte do ambiente é importante no planejamento agrícola e ambiental para o correto dimensionamento das obras, tanto na construção civil quanto na conservação do solo (VIEIRA \& CARVALHO, 2001).

\footnotetext{
${ }^{1}$ Trabalho financiado pela EMBRAPA.

${ }^{2}$ Ph.D. em Estatística, Pesquisador, Embrapa Informática Agropecuária, Caixa Postal 6041, Campinas - SP, jruy@ cnptia.embrapa.br

${ }^{3}$ Doutor em Hidrologia e Matemática, Pesquisador, Embrapa Informática Agropecuária, Campinas - SP.

Recebido pelo Conselho Editorial em: 24-6-2003

Aprovado pelo Conselho Editorial em: 22-3-2005
} 
Inúmeros métodos de interpolação, com diversos níveis de complexidade, estão disponíveis na literatura (GOOVAERTS, 1999; CARVALHO et al., 2002). Como os métodos de krigagem usam a dependência espacial entre amostras vizinhas, expressa no semivariograma, para estimar valores em qualquer posição dentro do campo, sem tendência e com variância mínima, ou seja, são estimadores ótimos, este trabalho irá avaliar a krigagem ordinária. Esse método geoestatístico de interpolação é muito usado no estudo da distribuição espacial de precipitação pluviométrica. A correlação espacial entre observações vizinhas para predizer valores em locais não-amostrados é o aspecto fundamental que diferencia os interpoladores geoestatísticos dos demais.

O objetivo deste trabalho é apresentar método de comparação de interpoladores univariados usados na obtenção da distribuição espacial da precipitação pluvial média anual para o Estado de São Paulo.

\section{MATERIAL E MÉTODOS}

Para definir a metodologia de interpolação, foram utilizadas 1.027 observações provenientes de postos pluviométricos, representando todo o Estado.

Para a definição do estimador de krigagem ordinária, consideremos a situação de estimar o valor de um atributo contínuo $\mathrm{z}$ para qualquer local não-amostrado $\mathrm{u}$, usando os dados de $\mathrm{z}$ que foram amostrados na área de estudo $\boldsymbol{A}$. Krigagem é o nome genérico adaptado pelos geoestatísticos para a família de algoritmos de regressão de mínimos quadrados generalizados (GOOVAERTS, 1997; CARVALHO \& VIEIRA, 2001). Todos os estimadores de krigagem são variantes do estimador básico de regressão linear $Z(\mathbf{u})$, que é definido por:

$$
\mathrm{Z}(\mathbf{u})-\mathrm{m}(\mathbf{u})=\sum_{\alpha=1}^{n(u)} \lambda_{\alpha}(\mathbf{u})\left[\mathrm{Z}\left(\mathbf{u}_{\alpha}\right)-\mathrm{m}\left(\mathbf{u}_{\alpha}\right)\right]
$$

em que, $\lambda_{\alpha}(\mathbf{u})$ são os pesos definidos para os dados $\mathrm{z}\left(\mathbf{u}_{\alpha}\right)$, interpretado como realização da variável aleatória $Z\left(\mathbf{u}_{\alpha}\right)$. As quantidades $\mathrm{m}(\mathbf{u})$ e $\mathrm{m}\left(\mathbf{u}_{\alpha}\right)$ são os valores esperados das variáveis aleatórias $\mathrm{Z}(\mathbf{u})$ e $\mathrm{Z}\left(\mathbf{u}_{\alpha}\right)$. O número de amostras necessárias para a estimação bem como seus pesos variam de um local para outro. Na prática, somente os $\mathrm{n}(\mathbf{u})$ pares de observação perto do local $\mathbf{u}$ a ser estimado são mantidos. A interpretação de $\mathrm{z}(\mathbf{u})$ e $\mathrm{Z}\left(\mathbf{u}_{\alpha}\right)$, como realizações das variáveis aleatórias $\mathrm{Z}(\mathbf{u})$ e $\mathrm{Z}\left(\mathbf{u}_{\alpha}\right)$, permite a definição do erro de estimação como variável aleatória $Z(\mathbf{u})$ - $Z(\mathbf{u})$. A estimação por krigagem baseia-se na minimização da estimativa da variância do erro $\sigma_{\mathrm{E}}^{2}(\mathbf{u})$ sobre a suposição da não-tendenciosidade do estimador, ou seja:

$$
\sigma_{E}^{2}(\mathbf{u})=\operatorname{Var}\{Z(\mathbf{u})-Z(\mathbf{u})\}
$$

que é minimizada por:

$$
\mathrm{E}\{\mathrm{Z}(\mathbf{u})-\mathrm{Z}(\mathbf{u})\}=0
$$

Na krigagem ordinária, as flutuações locais da média são consideradas, limitando o domínio de sua estacionaridade para a vizinhança do local $\mathrm{W}(\mathbf{u})$, sendo a média constante, mas desconhecida. $\mathrm{O}$ estimador linear é definido por:

$$
\begin{aligned}
& \mathrm{Z}_{\mathrm{OK}}(\mathbf{u})=\sum_{\alpha=1}^{n(u)} \lambda_{\alpha}{ }^{\mathrm{OK}}(\mathbf{u}) \mathrm{Z}\left(\mathbf{u}_{\alpha}\right) \text { para } \\
& \sum_{\alpha=1}^{n(u)} \lambda_{\alpha}{ }^{\mathrm{OK}}(\mathbf{u})=1
\end{aligned}
$$

Os n(u) pesos $\lambda_{\alpha}{ }^{\mathrm{OK}}(\mathbf{u})$ são determinados de tal maneira que a variância do erro seja mínima. 
O inverso do quadrado da distância é um interpolador de médias ponderadas que não é exato. Quando o ponto de uma malha é calculado, os pesos atribuídos aos pontos são fracionários, porém sua soma é um. Se qualquer observação é coincidente com um ponto da malha, a distância entre essa observação e o ponto é zero com peso um, enquanto para as demais observações o peso é zero. É muito usado em diversas situações por ser um método rápido de interpolação (ISAAKS \& SRIVASTAVA, 1989). Sua equação é dada por:

$$
\mathrm{Z}(\mathbf{u})=\left(\sum_{\alpha=1}^{n(u)} \mathrm{z}\left(\mathbf{u}_{\alpha}\right) / \mathrm{h}_{\alpha}{ }^{2}(\mathbf{u})\right) /\left(\sum_{\alpha=1}^{n(u)} 1 / \mathrm{h}_{\alpha}{ }^{2}(\mathbf{u})\right)
$$

em que, $\mathrm{h}_{\alpha}$ é a distância entre os pares de observação $\mathrm{n}(\mathbf{u})$.

Curvatura mínima é um interpolador muito usado em ciências da terra. A superfície gerada pelo método de curvatura mínima é semelhante a um disco que passa por meio dos valores observados com pequena curvatura. Ela não é um interpolador exato e são necessários quatro passos para que a malha final seja gerada: $1^{\underline{0}}$ - uma regressão de mínimos quadrados é ajustada às observações; $2^{2}$ - os resíduos são calculados; $3^{\underline{0}}$ - o modelo de curvatura mínima é usado para interpolar os resíduos nos nós da malha de observação, e $4^{\underline{0}}$ - os valores do modelo de regressão nos nós da malha são adicionados nos resíduos interpolados, resultando na superfície final.

O algoritmo de curvatura mínima gera uma superfície que interpola todas as observações disponíveis por meio da solução da equação diferencial modificada biarmônica com tensão (SMITH \& WESSEL, 1990):

$$
\left(1-\mathrm{T}_{\mathrm{i}}\right) \nabla^{2}\left(\nabla^{2} \mathrm{Z}\right)\left(\mathrm{T}_{\mathrm{i}}\right) \nabla^{2} \mathrm{Z}=0
$$

com três conjuntos de condições-limite:

nas extremidades $\Rightarrow\left(1-\mathrm{T}_{\mathrm{i}}\right) \partial^{2} \mathrm{Z} / \partial \mathrm{n}^{2}+\mathrm{T}_{\mathrm{i}} \partial \mathrm{Z} / \partial \mathrm{n}=0$

$$
\partial\left(\nabla^{2} \mathrm{Z}\right) / \partial \mathrm{n}=0
$$

nos cantos $\Rightarrow \partial^{2} \mathrm{Z} / \partial \mathrm{x} \partial \mathrm{y}=0$

em que,

$\nabla^{2}$ - operador de Laplace;

$\mathrm{n}$ - fronteira normal, e

$\mathrm{T}$ - tensão e $\mathrm{Z}$ as observações.

Para comparar os três procedimentos de interpolação: inverso do quadrado da distância, curvatura mínima e krigagem ordinária, o método do Quadrado Médio do Erro - QME (ADDINK \& STEIN, 1999; PHILLIPS et al., 1992) será calculado pela seguinte formula:

$$
\mathrm{QME}=\left\{\sum_{\alpha=1}^{n(u)}\left(\mathrm{Z}_{\text {est }, \alpha}-\mathrm{Z}_{\alpha}^{*}\right)^{2}\right\} / \mathrm{n}(\mathbf{u})
$$

Os resultados obtidos para o quadrado médio do erro serão comparados e conclusões serão obtidas. O método que apresentar o menor quadrado médio residual será o método considerado como o mais eficiente.

\section{RESULTADOS E DISCUSSÃO}

Os dados anuais de precipitação são médias de 40 anos para o período de 1957 a 1997. Todas as análises foram realizadas nessas médias sem se preocupar com as flutuações anuais de um ano para o outro. Foram utilizadas para as análises geoestatísticas as rotinas desenvolvidas em Fortran por VIEIRA et al. (2002). Na Tabela 1, apresentam-se as estatísticas básicas da variável em estudo. 
TABELA 1. Estatísticas para a precipitação pluvial média anual em 1.027 postos pluviométricos.

\begin{tabular}{ccccc}
\hline \multicolumn{5}{c}{ Precipitação Pluvial Média Anual (mm) } \\
\hline Média & Desvio-Padrão & Mínimo & Máximo & C.V. (\%) \\
\hline $1.490,64$ & 304,92 & $1.077,70$ & $4.378,00$ & 20,46 \\
\hline
\end{tabular}

Como se verifica na Tabela 1, o coeficiente de variação (C.V.) pode ser considerado moderado; essa variação existente caracteriza-se, principalmente, pelos postos localizadas no litoral, cujos dados, devido a peculiaridades climáticas, não seguem a mesma tendência dos demais. Também foram obtidos os coeficientes de assimetria $(3,61)$ e curtose $(18,61)$, indicando que os dados não podem ser considerados normalmente distribuídos (assimetria $=0$ e curtose $=3$ ).

O semivariograma experimental para a precipitação média anual é apresentado na Figura 1, sendo utilizado para avaliar a dependência espacial da variável em estudo. Visando a tornar mais homogêneos os dados do litoral e do interior, a transformação de logaritmo neperiano foi usada. FIGURA 1. Precipitação pluvial média anual em mm. Modelo esférico ajustado.

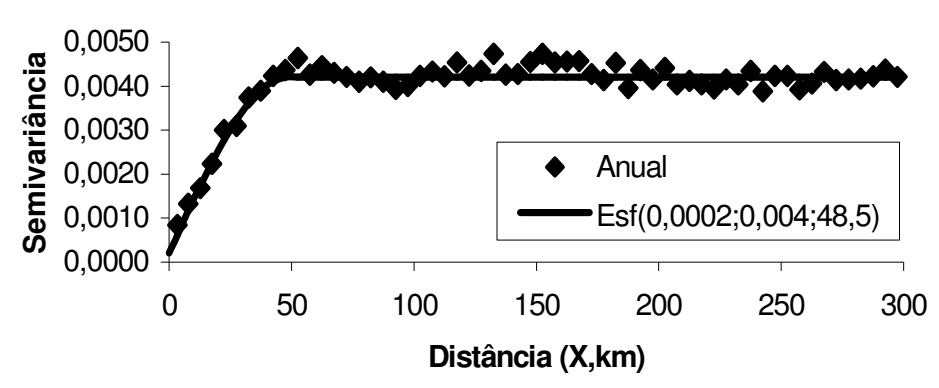

O grau de ajuste do modelo foi verificado por meio do coeficiente de determinação $\mathrm{R}^{2}=0,95$, mostrando o quanto o modelo de regressão explica a variabilidade total da variável em estudo. A validação desses modelos foi realizada por meio do procedimento de auto-validação "Jack-Knifing" (VIEIRA, 2000). O exame do semivariograma para a precipitação média anual revela que existe dependência espacial, em que o efeito pepita é $\mathrm{C}_{0}=0,0002$ e o alcance a $=48,5 \mathrm{~km}$. O efeito pepita de 0,0002 significa que existe descontinuidade entre valores separados por distâncias menores do que a usada no intervalo de amostragem. A proporção desse valor para o patamar do semivariograma $\left(\mathrm{C}_{0}+\right.$ $\mathrm{C}_{1}$ ), no caso $4,76 \%$, é indicativo da quantidade de variação ao acaso de um ponto para outro, e quanto menor seu valor, mais parecidos são os valores vizinhos. O alcance (a) de 48,5 km significa que todos os vizinhos dentro desse raio podem ser usados na estimativa de valores em espaçamentos mais próximos. Os semivariogramas direcionais, na Figura 2, mostram que as variabilidades podem ser consideradas idênticas em todas as direções $\left[0^{\circ}\right.$ (direção do eixo x); $45^{\circ} ; 90^{\circ}$ e $-45^{\circ}$ ] escolhidas na área experimental, apresentando, portanto, isotropia.

Como a dependência espacial para a variável em estudo foi obtida, a krigagem ordinária foi usada para estimar valores em pontos não-amostrados. Os valores obtidos por meio da krigagem são não-viciados, têm variância mínima (GOOVAERTS, 1997; VIEIRA, 2000) e são ideais para a construção de mapas de isolinhas ou tridimensionais para a verificação e a interpretação da variabilidade espacial. As informações mostradas nesses gráficos de isolinhas são muito úteis para entender a variabilidade das propriedades da precipitação pluvial e para identificar áreas que necessitam de maiores ou menores cuidados, como pode ser visto na Figura 3, com as observações retransformadas para os padrões originais. 


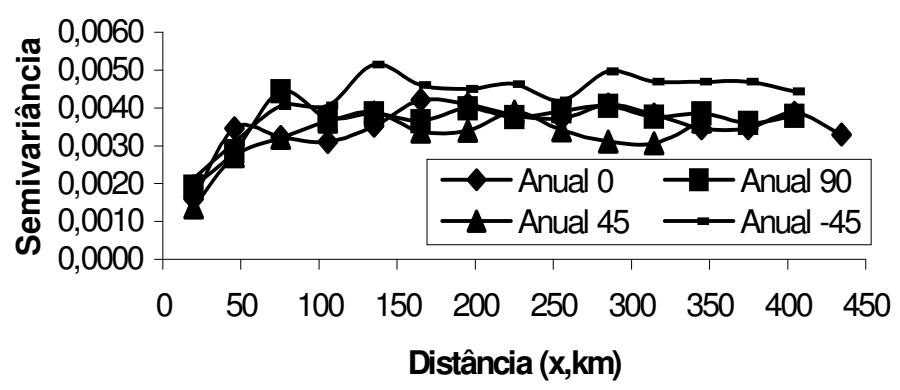

FIGURA 2. Semivariogramas Direcionais para precipitação pluvial média anual no Estado de São Paulo. Direções usadas $0^{\circ}$ (direção do eixo x); $45^{\circ} ; 90^{\circ}$ e $-45^{\circ}$.

Na Figura 3, obtida por meio do uso do software Surfer - versão 7.04 (Golden Software Inc., 2001), mostra-se uma faixa de alta precipitação pluviométrica no litoral do Estado. Os índices pluviométricos dos postos localizadas no litoral, devido ao relevo da região, apresentam resultados que seguem ordem própria, sendo discrepantes dos demais. Isso ocorre devido ao relevo concordante (a Serra do Mar se dispõe mais ou menos paralelamente à linha da costa), as vertentes a barravento (lado de onde sopra o vento) são mais expostas aos ventos úmidos. O ar depara-se com a barreira montanhosa e é obrigado a subir. Ao subir, arrefece e satura, dando-se a condensação do vapor de água e a formação de nuvens originando a queda de precipitação do tipo orográfico ou de relevo. As vertentes opostas são mais protegidas pelos ventos, e o ar é mais seco, pelo que a distribuição de precipitação é mais constante.

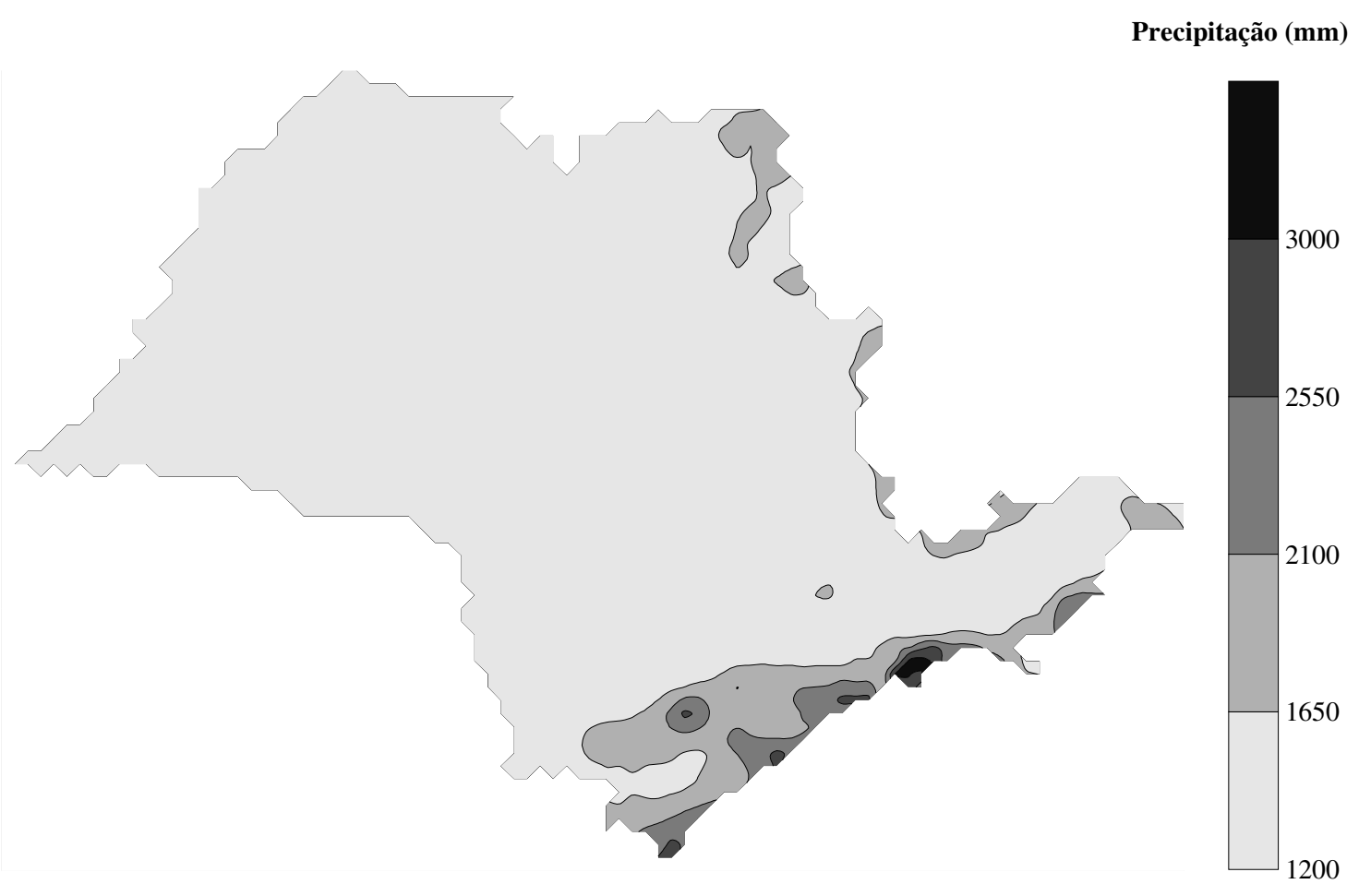

FIGURA 3. Distribuição espacial para precipitação pluvial média anual no Estado de São Paulo obtida por meio de krigagem ordinária. 
Com o objetivo de comparar o interpolador de krigagem ordinária com os interpoladores de inverso do quadrado da distância e curvatura mínima, a distribuição espacial para a precipitação média anual foi calculada, conforme mostrado nas Figuras 4 e 5.

Precipitação (mm)

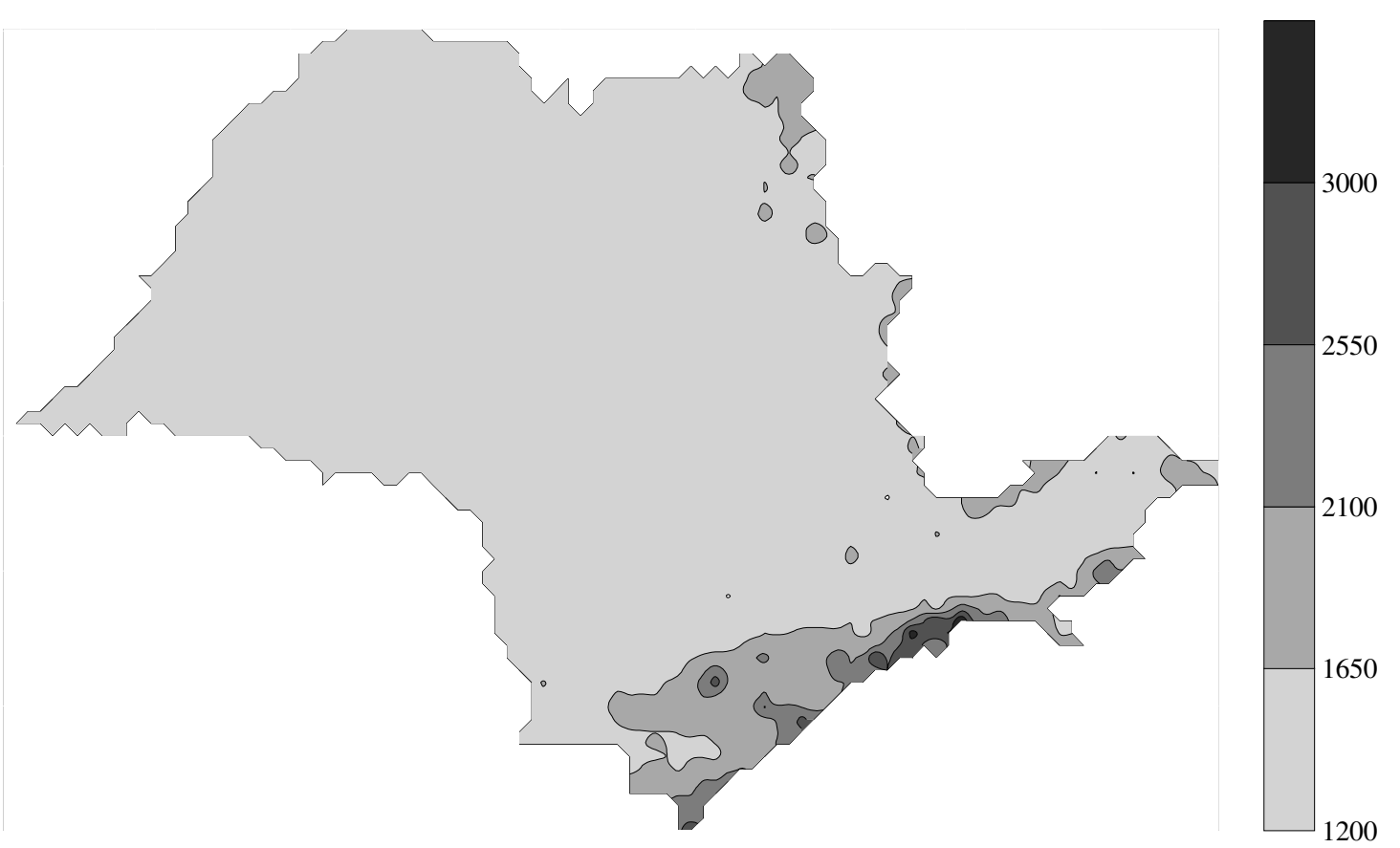

FIGURA 4. Distribuição espacial para precipitação pluvial média anual no Estado de São Paulo obtida por meio do inverso do quadrado da distância.

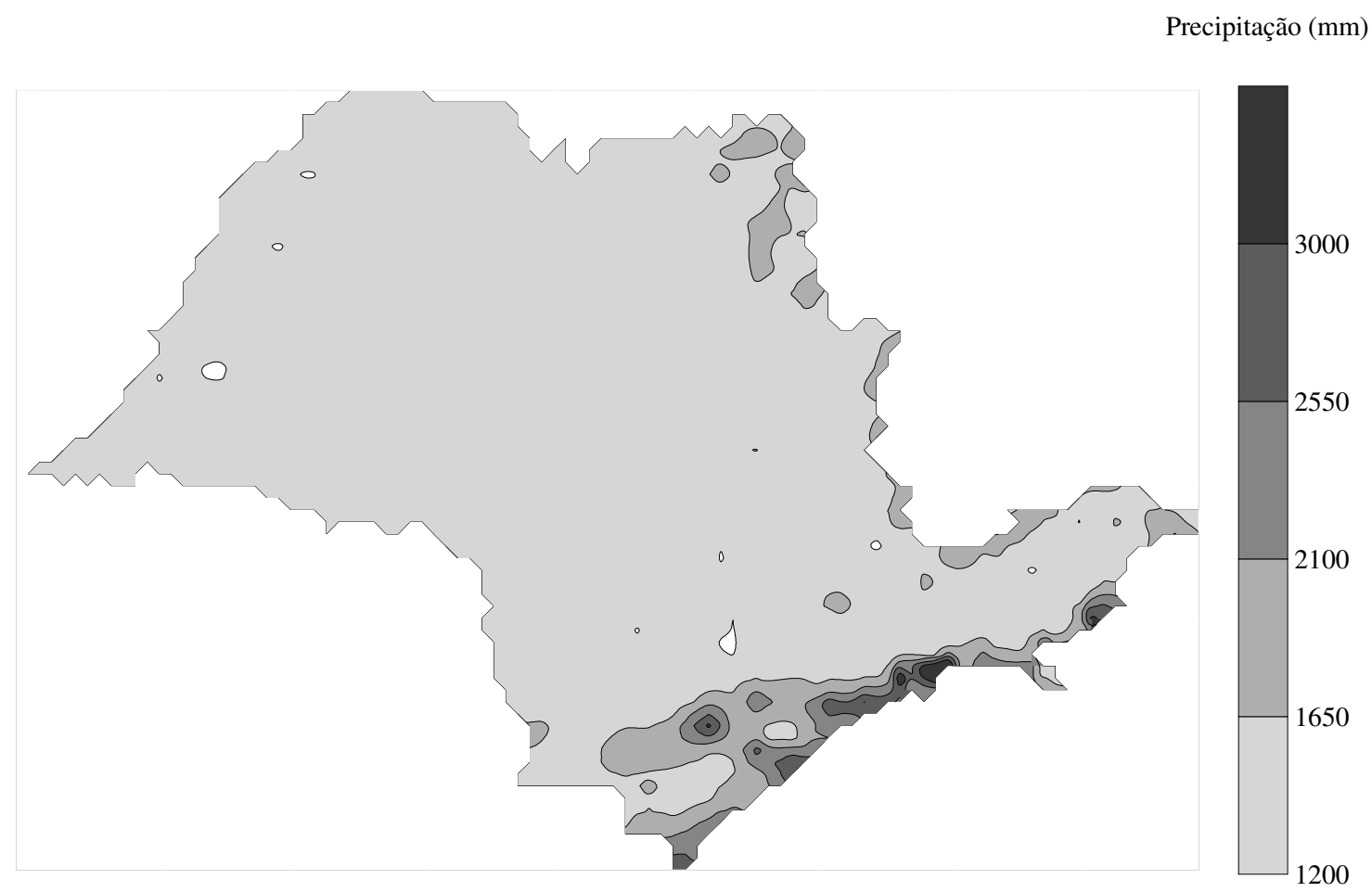

FIGURA 5. Distribuição espacial para precipitação pluvial média anual no Estado de São Paulo obtida por meio da curvatura mínima. 
Pela comparação dos mapas obtidos, verifica-se que o método de krigagem ordinária apresenta distribuição espacial muito mais homogênea do que os demais. Esse resultado é obtido principalmente pelo fato de esse interpolador ser não-viciado, com variância mínima, ou seja, é um interpolador ótimo.

O desempenho desses interpoladores foi obtido e comparado usando o critério do quadrado médio do erro. Para os valores estimados pelos métodos do inverso da distância ao quadrado e curvatura mínima, esse critério pode ser aplicado diretamente, pois não são interpoladores ótimos, e a diferença dos valores estimados e os observados não é nula. Entretanto, para o interpolador de krigagem ordinária, por ser ótimo, essa diferença é nula, logo, para ter os valores estimados no ponto observado, o método de validação cruzada (VIEIRA, 2000; ISAAKS \& SRIVASTAVA, 1989) foi usado. Esse método envolve a estimativa de cada ponto medido, "fazendo de conta" que ele não existe durante a sua estimativa. A razão é que krigagem ordinária é um interpolador exato, passando exatamente pelo ponto observado, quando esse é usado no cálculo.

Na Tabela 2, apresentam-se os resultados obtidos para o critério de comparação QME. O valor do critério deve ser próximo de zero se o algoritmo é preciso. Altos erros estimados são obtidos para os três interpoladores. Para os dados em questão, o interpolador que apresentou resultado mais acurado, foi o de krigagem ordinária, seguido por curvatura mínima e, por último, o inverso do quadrado da distância.

TABELA 2. Valores do quadrado médio do erro para interpoladores usados na distribuição espacial de precipitação pluvial média anual.

\begin{tabular}{ccc}
\hline Krigagem Ordinária & Inverso do Quadrado da Distância & Curvatura Mínima \\
\hline $24.952,80$ & QME & \\
\hline
\end{tabular}

\section{CONCLUSÕES}

Os resultados confirmam que o interpolador geoestatístico de krigagem ordinária, por ser estatisticamente ótimo, apresenta melhor resultado que os demais interpoladores que ignoram a dependência espacial entre observações, usados neste estudo.

Os postos pluviométricos situados no litoral do Estado apresentam distribuição de precipitação pluviométrica diferente dos demais postos.

As observações são espacialmente dependentes até um alcance de 48,5 km, em todas as direções.

\section{REFERÊNCIAS}

ADDINK, E.A.; STEIN, A. A comparison of conventional and geostatistical methods to replace cluoded pixels in NOAA-AVHRR images. International Journal of Remote Sensing, Dundee, v.20, n.5, p.961-77, 1999.

CARVALHO, J.R.P. de; VIEIRA, S.R. Avaliação e comparação de estimadores de krigagem para variáveis agronômicas - uma proposta. Campinas: Embrapa Informática Agropecuária, 2001. 21 p. (Documentos, 9)

CARVALHO, J.R.P de; SILVEIRA, P.M. da; VIEIRA, S.R. Geoestatística na determinação da variabilidade espacial de características químicas do solo sob diferentes preparos. Pesquisa Agropecuária Brasileira, Brasília, v.37, n.8, p.1151-9, ago. 2002.

GOLDEN SOFTWARE INC. Surfer version 7.04. Golden, 2001. 
GOOVAERTS, P. Geostatistics for natural resources evaluation. New York: Oxford University Press, 1977. $483 \mathrm{p}$.

GOOVAERTS, P. Performance comparison of geostatistical algorithms for incorporating elevation into the mapping of precipitation. GeoComputation, 1999. p.1-18. Disponível em: <http://www.geovista.psu.edu/sites/geocomp99/Gc99/023/gc_023.htm>. Acesso em: 7 abr. 2004.

ISAAKS, E.H.; SRIVASTAVA, R.M. Applied geostatistics. New York: Oxford University Press, 1989. $561 \mathrm{p}$.

PHILLIPS, D.L.; DOLPH, J.; MARKS, D.A comparison of geostatistical procedures for spatial analysis of precipitations in mountainous terrain. Agricultural and Forest Meteorology, Amsterdam, v.60, n.1-2, p.119-41, aug. 1992.

SMITH, W.H.F.; WESSEL, P. Gridding with continuous curvature splines in tension. Geophysics, v.55, n.3, p.293-305, 1990.

VIEIRA, S.R. Geoestatística em estudos de variabilidade espacial do solo. In: NOVAIS, R.F. de; ALVAREZ V.H.; SCHAEFER, C.E.G.R. Tópicos em ciência do solo, Viçosa - MG: Sociedade Brasileira de Ciência do Solo, 2000. v.1, p.1-54.

VIEIRA, S.R.; CARVALHO, J.R.P. de. Estudo da periodicidade temporal de chuvas em bacia hidrográfica dos Rios Turvo/Grande - uma proposta. Campinas: Embrapa Informática Agropecuária, 2001. 17 p. (Documentos, 10).

VIEIRA, S.R.; MILLETE, J.; TOPP, G.C.; REYNOLDS, W.D. Handbook for geostatistical analysis of variability in soil and meteorological paramaters. In: ALVAREZ V.H. Tópicos em Ciência do Solo II. Viçosa - MG: Sociedade Brasileira de Ciência do Solo, 2002. v.2, p.1-45. 